\title{
El cadete Agustín Melgar y la atención del trauma vascular periférico militar durante la Batalla de Chapultepec
}

\author{
Cadet Agustín Melgar and military peripheral vascular trauma approach during \\ Chapultepec's Battle
}

Luis M. García-Núñez*, Gabriela A. Guillén-Hernández, Edgar F. Hernández-García, Oscar E. Pérez-Morales, Raúl García-Ramírez y Fernando F. Arcaute-Velázquez

Departamento de Cirugía General, Hospital Central Militar, Secretaría de la Defensa Nacional, Ciudad de México, México

\begin{abstract}
Resumen
El trauma del órgano vascular periférico es una condición muy frecuente en los conflictos militares. Por fortuna, la comprensión integral de la fisiopatología local y sistémica, en adición al desarrollo de técnicas quirúrgicas innovadoras y avances tecnológicos, ha mejorado el pronóstico relativo a la sobrevida y la conservación anatómica y funcional de las extremidades. En este trabajo se hace una revisión histórica y del estado actual del abordaje del trauma vascular periférico, sobre la base de un episodio heroico de la Campaña por la Ciudad de México durante la invasión norteamericana de 1846-1848: la defensa del Castillo de Chapultepec por el cadete Agustín Melgar.
\end{abstract}

Palabras Clave: Órgano vascular periférico. Pronóstico. Trauma.

\begin{abstract}
Trauma to peripheral vascular organ is a very frequent condition during military conflicts. Fortunately, comprehensive understanding of local and systemic pathophysiology, in addition to the development of innovative surgical techniques and technological advances, have improved the outcome regarding to survival and anatomic and functional conservation of the limbs. In this manuscript, we perform an historical and state of the art review related to the approach of the peripheral vascular trauma, on the basis of an heroical episode from the Mexico City's Campaign, during North American invasion 1846-1848: the defense of Chapultepec's Castle by cadet Agustin Melgar.
\end{abstract}

Key Words: Peripheral vascular organ. Outcome. Trauma.

\footnotetext{
Correspondencia:

*Luis M. García-Núñez

Enrique Sada Moguerza, 17, Torre B

Circuito Centro Comercial, Ciudad Satélite

C.P. 53100, Naucalpan de Juárez, Edo. de México, México

Fecha de recepción: 20-05-2018

Fecha de aceptación: 08-10-2019

E-mail: Imgarcian@ hotmail.com

DOI: $10.24875 /$ CIRU. 19000473

0009-7411/@ 2019 Academia Mexicana de Cirugía. Publicado por Permanyer. Este es un artículo open access bajo la licencia CC BY-NC-ND (http://creativecommons.org/licenses/by-nc-nd/4.0/).
} 


\section{Introducción}

Fue la mañana del 12 de septiembre de 1847 cuando el ejército norteamericano inició un feroz bombardeo sobre el Castillo de Chapultepec, sede del Colegio Militar y último bastión defensivo del ejército mexicano para evitar la toma de la Ciudad de México, durante la Intervención Norteamericana que se suscitó entre 1846 y 1848 . A las 9:20 horas del 13 de septiembre, con el desgaste físico y moral de los defensores ( 57 cadetes y poco más de 800 militares profesionales), impuesto por más de 15 horas de ataque con explosivos de artillería, casi 5,000 soldados enemigos iniciaron el asalto al fuerte escalando por las laderas del Cerro del Chapulín, formación rocosa volcánica en cuya cúspide se encontraba el castillo. Al llegar a sus patios encontraron una inusual resistencia armada por los jóvenes cadetes, estudiantes militares de entre 13 y 19 años. Tras 40 minutos de lucha y el aniquilamiento o la captura cuasi plenos de los defensores, el Castillo de Chapultepec fue tomado y la Bandera Nacional asegurada como trofeo de guerra. Así, a las 10:00 h de la mañana del 14 de septiembre de 1847, el pabellón de las barras y las estrellas ondeaba en el asta del Palacio Virreinal (hoy Palacio Nacional) 1 .

Destaca en esta jornada bélica la participación del cadete Agustín Melgar, uno de los alumnos del Colegio Militar que, junto con el teniente Juan de la Barrera y sus compañeros Fernando Montes de Oca, Francisco Márquez, Vicente Suárez y el soldado Juan Escutia, se conocen colectivamente en la historia oficial de México como «los Niños Héroes de Chapultepec». Estudiante del Arma de Caballería, de 19 años y originario de la Ciudad de Chihuahua, había reingresado pocos días antes del asalto al castillo, tras abandonar temporalmente la carrera de las armas. El 13 de septiembre, al inicio de la contienda, embravecido al observar cómo los enemigos asesinaban a sus compañeros, emprendió una titánica lucha con su fusil bayoneta. Narra la crónica de dos testigos, los sargentos Teófilo Noris e Ignacio Molina, que Melgar hirió y mató a varios contrincantes antes de refugiarse en la biblioteca del colegio. Ahí construyó un parapeto con muebles y colchones de paja, desde donde continuó la defensa. Cuando se quedó sin municiones, inició la lucha cuerpo a cuerpo con la bayoneta, viéndose superado numéricamente por los soldados enemigos y sufriendo heridas por arma blanca en el miembro torácico izquierdo y el muslo derecho, sangrando torrencialmente. Privado de la consciencia, en un acto humanitario sus rivales lo trasladaron al Hospital de Sangre de la fortaleza, donde por falta de médicos mexicanos un cirujano norteamericano amputó algunas horas después las extremidades lesionadas, al observar su estado gangrenoso y en un intento por controlar el sangrado y salvar su vida. Fue curado y vendado, dejándolo en una mesa para su recuperación. Al otro día, durante la evaluación de los daños del castillo, fue encontrado muerto en el suelo, con los vendajes arrancados, en clara manifestación de ardor por continuar con la pelea. Su bravura y valentía le valieron el reconocimiento de su grado y nombre por parte del Mayor Charles Winslow Elliot en los Partes de Guerra Americanos².

\section{Evaluación y tratamiento del cadete Agustín Melgar}

Es notable la humanidad con que los enemigos se ocuparon del cadete Melgar, aunque también es menester reconocer que su heroísmo condujo al Mayor Elliot a prestarle auxilio. Tras ser herido con bayonetas, Melgar fue llevado al Hospital de Sangre del Colegio Militar, donde no había médicos mexicanos, ya que el facultativo asignado al establecimiento militar era el Dr. Rafael Lucio, quien se reportó enfermo ese día y que en indagatorias de la posguerra manifestó incluso "...Que no tenía la obligación de asistir ese día...». Lo anterior fue causa de que después de su lesión transcurrieran varias horas antes de que un cirujano del ejército norteamericano evaluara las lesiones penetrantes que había sufrido el militar mexicano, y se instituyera el tratamiento pertinente para la época y el ámbito de campaña ${ }^{1,2}$.

Según lo que es posible interpretar de los documentos estadounidenses, cuando se prestó auxilio quirúrgico a Melgar persistía un sangrado activo y ya había gangrena en sus extremidades. Con mucha seguridad, el cirujano apreció falta de viabilidad y sangrado por algunas heridas penetrantes de carácter devastador, y decidió practicar las amputaciones para descontinuar la hemorragia, evitar la infección y salvar su vida. Desde esa época se sabía que la «falta de circulación de los fluidos nutritivos» y la «indisposición de los tejidos para la vida" generaban necrosis tisular, y que se requería amputar los miembros afectados para favorecer la sobrevida. Aunque por mala fortuna en las fuentes documentales militares no están descritos los procedimientos con los que se llevaron a cabo las amputaciones, es posible deducir que se 
practicaron en un teatro quirúrgico lejos de lo ideal, siguiendo las normas técnicas usuales para la época, procedentes de la experiencia bélica británica, empleando sujeción vigorosa del paciente por los asistentes médicos, analgesia con agentes ingeridos (en el mejor de los casos), uso de un torniquete compresivo, tajo y aserramiento de la extremidad siguiendo los límites anatómicos definidos para cada tipo de amputación, coagulación térmica con ignición y colocación de vendajes con vino o azúcar fundido. Es casi certero que no precedió intento alguno de reanimación del cadete, tanto por la falta de conocimiento de la importancia de la mejora de la hemodinamia de los pacientes traumatizados como por la premura con la cual el personal militar enemigo debía reiniciar las actividades de campaña ${ }^{3}$.

Aunque la narrativa histórica pretende adjudicar como motivo del arrancamiento de los vendajes y del sitio donde Melgar fue encontrado muerto el 14 de septiembre de 1847 (debajo de la mesa del procedimiento en la biblioteca del Colegio Militar) la «...clara manifestación de ardor por continuar con la pelea...", se sabe que el procedimiento quirúrgico de la amputación inflingía un intenso dolor en el paciente. Por tanto, es posible que el cadete Melgar, ya víctima de un estado coagulopático, sufriera grandes dolores, y que en su agitado intento por buscar ayuda y mitigar la sintomatología local se retirara los vendajes, resangrando y cayendo de la mesa, lo cual contribuyó a su deceso ${ }^{1,2}$.

\section{Descripción e interpretación histórica del caso clínico en términos modernos}

Al analizar lo anterior, es claro que Melgar fue víctima de traumatismos penetrantes del órgano vascular periférico por un instrumento de baja velocidad (bayoneta). Sin embargo, de acuerdo con los criterios de Johansen, descritos por Dawson, et al. ${ }^{4}$ y por García-Núñez, et al. ${ }^{5}$, las lesiones de esta naturaleza se clasifican técnicamente como «trauma vascular periférico complejo». En la cirugía del trauma posmoderna, estas lesiones se clasifican con la escala de la Asociación Americana para la Cirugía del Trauma (AAST-OIS, por las siglas de American Association for the Surgery of Trauma-Organ Injury Scale) en los grados I a V, con gravedad creciente. Sin embargo, hay que notar que la graduación depende de la localización de la lesión y no de su magnitud. Además, la escala también describe que hay que aumentar un grado para múltiples lesiones de grado III o IV que involucren más del $50 \%$ de la circunferencia del vaso, y disminuir un grado cuando haya laceraciones de menos del $25 \%$ de la circunferencia del vaso para lesiones de grado IV o $\mathrm{V}^{6}$.

Así, Melgar posiblemente sufrió lesiones arteriales de grado III o IV en el miembro torácico izquierdo (arteria axilar o braquial), combinadas con trauma venoso de grado III (venas axilar y braquial), mientras que en el muslo derecho pudo haber sufrido lesiones arteriales de grado IV o V (arteria femoral común, superficial o profunda) y trauma venoso de grado III (vena femoral común, superficial o profunda), por lo que en caso de haber sufrido solo estos daños se estiman 43 puntos en la Escala de Gravedad de la Lesión (ISS, Injury Severity Scale), definiendo un alto índice de gravedad anatómica. Se sabe que en los pacientes con trauma del órgano vascular periférico las lesiones arteriovenosas combinadas y una puntuación ISS > 25 condicionan alta morbimortalidad, elevando 12.5 veces la posibilidad de sufrir complicaciones y 22.4 veces la de muerte ${ }^{6-11}$.

Aunque hay varios signos y síntomas que hacen sospechar la presencia de lesiones del órgano vascular periférico, el sangrado como principal manifestación clínica y causa más importante de muerte en estos casos, así como los datos obvios de isquemia de la extremidad, son lo que las hacen aparentes inmediatamente $\mathrm{e}^{5,8-10,12}$. La narrativa histórica apunta a que el cirujano norteamericano observó sangrado activo y datos de baja perfusión (estado gangrenoso) de las extremidades, lo cual hace más contundente la sospecha clínica que podemos establecer en la actualidad. Incluso en nuestros días, la conducta quirúrgica contemporánea recomienda ampliamente la amputación cuando la vida del paciente se encuentra en riesgo o hay datos de indiscutible inviabilidad de una extremidad lesionada.De esta manera, no hay cuestión alguna en que el procedimiento estuvo instituido bajo la normativa técnica que aún es vigente $\mathrm{e}^{3,13-16}$.

En términos pronósticos actuales, la amputación y la muerte son raras en las lesiones de los vasos axilares y braquiales, aun en presencia de lesiones nerviosas asociadas. Sin embargo, hay una alta tasa de morbilidad por los traumatismos de los vasos axilares. En las series militares actuales está descrito que se obtienen buenos resultados (completa resolución de la hipoperfusión y del déficit neurológico) en el $25 \%$ de los casos de trauma vascular axilar y en el $70 \%$ de los pacientes con lesiones vasculares braquiales ${ }^{8,9,13}$. En las víctimas de trauma vascular femoral, los índices pronósticos 
contemporáneos también están bien definidos. Aunque en la actualidad la tasa de mortalidad alcanza el $9 \%$ y la posibilidad de amputación se sitúa en el 3\%, la morbilidad puede alcanzar cifras de hasta el $55 \%$, figurando en ella la infección de las heridas, la trombosis arterial y venosa, el síndrome compartimental y la hemorragia perioperatoria ${ }^{7,9}$.

\section{Evolución en el abordaje del trauma vascular periférico militar}

Aunque la atención de las heridas es una evidente $y$ ancestral necesidad en el abordaje del trauma militar, fue con Ambroise Paré (siglo XVI) cuando se dieron los grandes avances operatorios en un conflicto armado, tratando un gran volumen de lesionados en condiciones austeras ${ }^{3,14-16}$.

El control del sangrado consecutivo a la lesión ha sido una gran preocupación desde el inicio de la Humanidad. Desde el año 1600 a.C. se usaron recursos como hilos vegetales, hierros y líquidos hirvientes, vendajes y compresión, que se describen en el Papiro de Eber. En las primeras dos centurias d.C., Celso describió el amarre (ligadura) de las fuentes de sangrado, mientras que la amputación prevaleció en los casos de extremidades gangrenosas, de las que se sabía, además, que podían ser fuente de hemorragia. Rufus de Éfeso, en el siglo I d.C., notó que las lesiones parciales de los ductos sanguíneos eran más difíciles de controlar que si se seccionaban por completo, lo que llevó a Galeno (siglo II) a advertir que, en el primer caso, colocar un dedo para presionar la herida conducía a la formación de un coágulo y la detención del sangrado. Paré, en 1552, con su amplia experiencia, recomendó la ligadura para la hemostasia en lesiones abiertas, empleando para asegurar de forma preliminar el vaso el bec de corbin, ancestro del moderno hemostato. Luego se reconoció la circulación colateral, lo cual permitió implementar la conducta observacional aun después de la ligadura de grandes estructuras vasculares, con la prudente esperanza de la conservación del miembro. Finalmente, también se adjudicó una gran importancia a la limpieza perioperatoria como factor de alto peso para evitar las infecciones, que en la mayoría de los casos eran mortales ${ }^{3,13-16}$.

La reparación arterial directa de una lesión militar fue descrita por Hallowell 243 años después de que Paré señalara la ligadura como la terapia de elección en estas condiciones. Luego, Broca (1762), Gluck (1883), Postempski (1886), Von-Horoch (1887), Jassinowsky y Brucci (1889), Muscatello
(1890), Heidenhain (1894), Sabanyeff (1896) y Murphy (1897) reportaron el éxito progresivo en la angiorrafia primaria, ya en conjunto con el empleo de la anestesia y la asepsia perioperatoria ${ }^{3,13-16}$.

En México, en el siglo XVI, Alonso López de Hinojosa, en su obra Summa y recopilación de Chirugia, con un arte para sangrar muy útil y provechoso, reportó la ligadura arterial por trauma o patología vascular, misma que se empleó durante todo el siglo XIX, sobre todo en las lesiones de campaña. Notable fue la contribución en este aspecto de Aronssohn y Montes de Oca, cuyos principios fueron seguidos por Pedro van der Linden, cirujano en jefe durante la campaña de la Ciudad de México en $1847^{17}$.

Fue también por Murphy (1897), del Hospital Cook de Chicago, que se practicó y describió la primera anastomosis termino-terminal. Carrel, en 1912, desarrolló la técnica de triangulación para las anastomosis vasculares, lo que es una contribución monumental en este rubro, empleándose hasta hoy en cirugía vascular y de trasplante ${ }^{18,19}$.En 1912, Guthrie describió por primera vez la colocación de un injerto venoso para puentear un defecto arterial, usando la técnica de Carrel. Matas (1913) apuntó la deformación arteriovenosa postraumática (fístulas arteriovenosas y pseudoaneurismas) acral como «un grave problema militar», y recomendó su corrección quirúrgica, previo examen de la anatomía específica de la malformación. Soubbotitch (1913) describió la experiencia militar en las guerras Serbo-Turca y Serbo-Búlgara, con una tasa impresionantemente baja de infección y resangrado. En la Primera Guerra Mundial, los cirujanos militares alemanes reportaron más de 100 casos de arteriorrafias exitosas, pero también, en 1915, con el desarrollo de nuevos y más poderosos explosivos de artillería, refinaron las técnicas de amputación, pues las reparaciones arteriales resultaron imprácticas, ya que los intentos de angiorrafia llevaban frecuentemente a resultados gangrenosos y hemorragia secundaria ${ }^{5,7,8}$.

En la Segunda Guerra Mundial, DeBakey y Simeone analizaron los resultados de la terapia de más de 2,000 lesiones arteriales, y encontraron que, aunque casi el $100 \%$ de los casos se ligaron, solo el $49 \%$ requirieron amputación. En los 81 casos sujetos a reparación arterial hubo una tasa de amputación del $35 \%$, mientras que con la colocación de injertos venosos esta tasa fue del $58 \%$. Sin embargo, después de la devastación del Día D, los autores concluyen que aunque no hay otro tratamiento más efectivo que la ligadura para las lesiones vasculares militares, definitivamente no es el procedimiento de elección, sino 
una técnica que tiene el propósito básico de detener el sangrado para permitir después estimar la localización, el tipo, el tamaño y el carácter de las lesiones en campaña, y ofrecer entonces el tratamiento definitivo más conveniente $e^{7-10,15,18}$.

En Corea, Jahnke y Selley (1953), Hughes (1955 y 1958) e Inui, Shanon y Howard (1955) reportaron significativas mejoras en los índices pronósticos de sobrevida, preservación y funcionalidad de los miembros, lo cual sin duda se debió al progreso sustancial en las técnicas de cirugía vascular y anestesia, al desarrollo de la angiografía, a la terapia transfusional y a los antibióticos, así como a la disponibilidad de aeronaves para el transporte rápido.En Vietnam, Rich (1968) reportó que, con el empleo de helicópteros, el 95\% de los heridos en campaña llegaban vivos a los hospitales quirúrgicos a pesar de la gravedad de sus lesiones. Con su experiencia, Rich y Hughes ${ }^{17-19}$ desarrollaron en 1970 el Reporte del Registro Vascular de Vietnam $(n=1000)$, en el que se observan ciertos aspectos esenciales que caracterizaron a la práctica quirúrgica. El método más frecuente para tratar a estos pacientes fue la interposición de injerto venoso autólogo (45.9\%), seguido por la anastomosis termino-terminal $(37.7 \%)$ y la angiorrafia lateral $(8.7 \%)$; solo 15 pacientes $(1.5 \%)$ se sometieron a ligadura vascular. La tasa de mortalidad fue del $1.7 \%$ y la tasa de amputación fue del $12.7 \%$, siendo la mayoría de ellos pacientes con lesiones vasculares poplíteas $7,8,10,13,17,18$.

Ya que en la actualidad los conflictos militares, en el contexto político de su definición, no son verdaderamente frecuentes, la experiencia civil es la que ha permitido continuar con la evolución del abordaje del trauma vascular. Mientras que algunas innovaciones pueden ser aplicables a la arena militar, otras no son útiles para el manejo de las lesiones castrenses. Además, es necesario reconocer que el carácter de los conflictos bélicos está sufriendo cambios dramáticos. Ahora las batallas no son tan frontales como antes y se están desarrollando artefactos militares altamente poderosos y letales, lo cual también seguramente modificará el patrón lesional vascular. Además, hay una tendencia a retirar de la línea del frente y desplazar a la retaguardia los servicios médicos, con el fin de conservar sus preciados recursos humanos y materiales. Esto afectará sin duda el abordaje del trauma vascular, ya que muchos cirujanos desplegados en el terreno de lucha no lograrán una experiencia vasta en el manejo del trauma en general, y mucho menos en el tratamiento apropiado del trauma vascular.
Aunado a la antes comentada falta de desarrollo de destrezas quirúrgicas, la disponibilidad de la radiología intervencionista y la última frontera de la robótica también ejercen un impacto en la solvencia de los cirujanos militares para manejar las lesiones vasculares. Sin duda alguna, la simulación y los modelos físicos ayudarán a cubrir estos déficits educativos, mejorando la calidad del desempeño de los cirujanos en el manejo de las lesiones militares del órgano vascular periférico $0^{8,16-19}$.

\section{Estándar moderno de tratamiento de las lesiones del órgano vascular periférico en la arena militar}

A continuación se comentan varios aspectos técnicos notables relativos a los estándares modernos de tratamiento en los pacientes con el perfil del cadete Agustín Melgar.

\section{Diagnóstico}

En los pacientes con lesiones militares del órgano vascular periférico la exploración física es esencial, ya que puede haber signos "duros» y «blandos» de lesión que definen la presencia de este tipo de traumatismos. El examen con dúplex puede auxiliar en la toma de decisiones, y cuando sea posible debe practicarse una arteriografía, aun en ambientes austeros. La angiotomografía es una herramienta a la que se ha reconocido un papel revolucionario en el diagnóstico de las lesiones del órgano vascular periférico ${ }^{8,10}$.

\section{Exanguinación}

En los pacientes con lesiones del órgano vascular periférico la «exanguinación» es una situación que demanda un mejor concepto, no solo clínico, sino también fisiológico y bioquímico. Aunque Asensio la definió en 2003 como «la más extrema forma de hemorragia, con una pérdida inicial de más del $40 \%$ del volumen circulante y sangrado activo a una tasa tal que, si no se controla rápido, causa la muerte del paciente", ahora hay gran conocimiento de los efectos celulares y subcelulares del sangrado y su continuum patológico ${ }^{20-22}$. La detección oportuna de su presencia, la comprensión de sus consecuencias y la rápida implementación de la terapia pertinente, incluyendo la institución temprana de la estrategia integral del control de daños, han probado impactar benéficamente sobre un aspecto celular de gran trascendencia 
clínica: atenuar la disminución en la ubiquitinación de la alfa-espectrina eritrocitaria, una proteína de soporte de la estructura celular que, cuando falla, conduce a una disminución en la rigidez y una deformidad de los eritrocitos en los lechos microvasculares durante el estado de choque, formación de microtrombos, hipoxia generalizada, falla orgánica múltiple y la muerte del paciente ${ }^{23}$.

\section{Reanimación en el terreno}

En la actualidad hay varios principios que rigen este rubro en las víctimas de trauma militar del órgano vascular periférico. La hidratación oral, tratando de conservar el estado de consciencia y el pulso radial, es útil en el ambiente austero. Otra alternativa, como la reanimación microvolumétrica con solución salina hipertónica, posee gran valor al poner en la balanza aspectos logísticos relativos al transporte de insumos, aunque su uso hospitalario no se ha difundido en la medida esperada. La transfusión con radios fijos en el campo de batalla, observando las proporciones que deben mantenerse entre los distintos derivados hemáticos, seguramente estará disponible en un futuro cercano, al mejorar la tecnología de conservación de estos productos biológicos 5 ,8-10.

\section{Scoop and run}

Es sabido que los mejores índices pronósticos se obtienen con el rápido transporte del paciente a un centro de trauma y su reanimación en ruta. Aunque el transporte aéreo es útil en pacientes con trauma vascular y otro tipo de lesiones devastadoras de la arena militar, en el trauma urbano solo beneficia a aquellos con un estado hemodinámico precario o que han sufrido paro cardiaco traumático. Así, es recomendable que todo paciente con trauma militar del órgano vascular periférico sea inmediatamente trasladado a un escalón quirúrgico, y reanimado durante su transporte, a fin de obtener las mejores posibilidades de sobrevida y recuperación funcional8,12,13.

\section{Control de daños}

- Reanimación de control de daños y control del sangrado. La primera intervención que debe hacerse en un paciente con trauma del órgano vascular periférico en la arena militar es cohibir el sangrado, haciendo uso de compresión manual o vendajes avanzados, con o sin hemostáticos tópicos. Luego, en el escalón quirúrgico, hay que implementar los principios modificados de la «hipotensión permisiva» y la «reanimación diferida», que aunque están diseñados como ejes rectores de la fluidoterapia avanzada en lesiones vasculares centrales, su uso juicioso puede beneficiar los índices pronósticos de estos casos, en ausencia de trauma craneoencefálico coexistente. La reanimación diferida se establece en pacientes con sangrado no controlado o parcialmente controlado que tienen acceso inmediato al quirófano, y consiste en mantener dos líneas venosas periféricas permeables listas para ser usadas. Por otro lado, la hipotensión permisiva se instituye si no hay recursos quirúrgicos inmediatamente disponibles, y con ella se pretende mantener una presión arterial media de 50-55 mmHg, a fin de evitar la dilución de los factores de la coagulación y expulsar con un alto gradiente de presión los coágulos formados en los vasos lesionados. Su buena práctica ha evidenciado que en los casos de lesiones vasculares centrales, sobre todo en las de naturaleza penetrante, se logra disminuir la cantidad de cristaloides infundidos y el volumen de hemoderivados administrados, aumentando la sobrevida en las primeras 24 horas, lo que permite instituir un mayor número de maniobras salvatorias para la vida ${ }^{4,5,8-13,15,19}$.

- Los shunts intravasculares temporales son dispositivos tubulares con un lumen que se colocan en el interior de un vaso lesionado para puentear los defectos de estas estructuras, controlar el sangrado rápidamente y mantener la perfusión distal, cumpliendo así con la premisa del control de daños. Se colocan con facilidad, no requieren anticoagulación sistémica, se mantienen permeables hasta por 1000 minutos y la irrigación del lecho distal puede recuperarse hasta en un $60 \%{ }^{5}$. Dawson y García Nuñez $z^{4,5}$ señalan que las lesiones militares del órgano vascular periférico representan una indicación ideal para el empleo de shunts intravasculares temporales, pues comúnmente coexiste inestabilidad esquelética, hay grave contaminación del lecho lesionado, el manejo quirúrgico definitivo por lo habitual demanda más que una reparación simple y es consumidor de tiempo, y la regla es que existan otras lesiones que requieran un manejo más urgente ${ }^{4,5}$.

- Sin duda, la ligadura vascular es una estrategia primaria del control de daños que, aunque no es la terapéutica de elección, permite detener el 
sangrado y favorece significativamente la sobrevida en los pacientes con trauma del órgano vascular periférico de naturaleza militar. Tras ello, según los postulados de DeBakey y Simeone, lo ideal es evaluar la anatomopatología de la lesión y practicar la terapia definitiva pertinente. Una alternativa a la ligadura, que a su vez es una recomendación condicionada a favor de la institución del control de daños, es la colocación de los mencionados shunts intravasculares temporales, los cuales, además de controlar el sangrado, presentan la ventaja de mantener en cierto grado la perfusión distal $\left.\right|^{7-10,15,18}$.

\section{Tratamiento quirúrgico definitivo de las lesiones militares del órgano vascular periférico}

- Angiorrafia primaria y angio-angioanastomosis: las reparaciones primarias pueden practicarse con sutura sintética inabsorbible monofilamento, con cualquier técnica estándar aceptada en la actualidad, si la anatomía de la lesión permite efectuar la rafia sin una estenosis superior al $50 \%$. En caso de no ser posible, se recomienda elegir una alternativa para evitar la morbilidad del cierre estenótico (trombosis, insuficiencia circulatoria, etc. $)^{7-12}$. Las anastomosis arteriales termino-terminales se practican si se interrumpe la integridad de la arteria y es posible unir los extremos con una técnica estándar. Esto puede no ser factible en muchos casos por la naturaleza lacerante y destructiva del trauma militar, dando paso a la interposición de un injerto. Debido a la gran posibilidad de trombosis en las anastomosis venosas, hay que ser juicioso al intentar su práctica, pues esto puede no contribuir a revascularizar la extremidad y ser consumidor de tiempo, elevando la mortalidad por agotamiento metabólico ${ }^{7-12}$.

- Empleo de injertos y parches: los parches vasculares (venosos) pueden colocarse para evitar el cierre estenosante en casos bien seleccionados. Sin embargo, las lesiones destructoras por lo general demandan la resección del vaso y interposición de un injerto. Los injertos venosos safenos reversos, obtenidos habitualmente de la extremidad contralateral cuando hay lesiones vasculares en los miembros inferiores, son una buena opción para interponer un injerto biológico, tienen alta disponibilidad y se recomienda su uso liberal siempre y cuando no comprometan la vascularización de la extremidad. Cuando se decide el empleo de injertos sintéticos, deben emplearse injertos anillados de calibre pertinente ${ }^{12}$.

- Ligadura: sin duda, la ligadura está perfilada como el tratamiento definitivo de una gran proporción de lesiones venosas periféricas en la arena militar, debido a la alta tasa de trombosis relacionadas con reparaciones más complejas que la simple venorrafia lateral. Por otro lado, aunque es deseable mantener la continuidad de los flujos arteriales, ya sea por arteriorrafias primarias o por colocación de injertos autólogos o sintéticos, no debe perderse de vista que conservar la vida es prioritario sobre preservar un miembro, pues procedimientos prohibitivamente prolongados y consumidores de tiempo incrementan la magnitud del second hit y modifican negativamente los índices pronósticos de sobrevida y funcionalidad ${ }^{4,5}$. En estos casos, la ligadura arterial queda plenamente justificada como intervención salvatoria, en el concepto de que, debido a la circulación colateral, se estima que solo un $35-50 \%$ de los pacientes con lesiones del órgano vascular periférico requerirán amputación, aunque la tasa de morbilidad funcional puede llegar a ser significativamente alta ${ }^{7-11,15,18}$.

- Técnicas adjuntas de salvamento de las extremidades: en estas lesiones pueden emplearse vasodilatadores intraarteriales, como papaverina o tolazolina, para evitar el espasmo. La anticoagulación con heparina de bajo peso molecular por lo general es segura cuando no hay lesiones asociadas, pero hay que vigilar estrechamente la aparición de complicaciones hemorragíparas. El empleo de dextrano de bajo peso molecular ( $500 \mathrm{ml}$ cada $12 \mathrm{~h}$ ) es un buen adyuvante, ya que induce un estado de anticoagulación relativo y mantiene permeables las reparaciones vasculares, sin predisponer gravemente al sangrado $0^{7-12}$. Aunque es poco frecuente que se coloquen endoprótesis en este tipo de lesiones, pueden ser un recurso muy útil en ciertos casos, principalmente en pacientes con fistulas arteriovenosas y pseudoaneurismas ${ }^{24-26}$.

\section{Síndrome de isquemia-reperfusión}

Si sangrar solo ocasionara estado de choque, esto sería muy fácil y esta aseveración aplica también con precisión a las víctimas de lesiones del órgano vascular periférico en la arena militar. Por desgracia, la 
hemorragia acarrea secuelas proinflamatorias y estas, a su vez, promueven que tras la reanimación se desarrolle el síndrome de isquemia-reperfusión, manifestado por alteraciones hidroelectrolíticas súbitas (hiperpotasemia, hipocalcemia) y cambios significativos en el balance ácido-base, causado por el lavado de ácidos orgánicos y de productos del metabolismo anaerobio en los lechos tisulares. Lo anterior lleva al paciente a arritmias y falla orgánica múltiple. La endoteliopatía postraumática conduce también al paciente a síndrome de fuga capilar, vasodilatación persistente y choque refractario a fluidos y hemotransfusión, lo que se traduce en una alta mortalidad $4,5,8-10,13,14,19-22$.

\section{Coagulopatía postraumática}

La coagulopatía postraumática es relativamente común en los pacientes con trauma del órgano vascular periférico en el ambiente austero de la arena militar. Los factores que promueven la aparición del fenómeno son el sangrado continuo, la exposición ambiental, las técnicas agresivas de reanimación con fluidos ( $\sin$ restricción volumétrica) y el retraso en el tratamiento definitivo. Por fortuna, en la actualidad, la combinación del rápido transporte, la reanimación limitada con fluidos en el terreno y durante la ruta de traslado, las políticas de fluidoterapia avanzada y hemoterapia temprana y protocolizada, así como la hemostasia farmacológica, han disminuido la incidencia de esta letal condición ${ }^{19-22}$.

\section{Síndrome compartimental}

En pacientes con lesiones del órgano vascular periférico, el síndrome compartimental de las extremidades se debe al aumento de la presión intersticial a un punto mayor que la presión capilar en un compartimiento fascial cerrado, lo cual causa compromiso del flujo sanguíneo y necrosis muscular y neural, lo que se asocia a graves consecuencias funcionales y para la vida. Aunque el tratamiento puede ser médico y conservador inicialmente, existen condiciones que demandan el tratamiento quirúrgico temprano, el cual en general consiste en la fasciotomía. Dichas condiciones son: 1) sexo masculino; 2) necesidades de altas cantidades de concentrados eritrocitarios para la reanimación del paciente; 3) fracturas expuestas; 4) heridas por proyectil de arma de fuego en las extremidades, principalmente si son de alta velocidad; 5) puntuación en la escala ISS > 16; y 6) edad mayor de 55 años.
La libre evolución del síndrome compartimental lleva a toxicidad sistémica, cambios gangrenosos, falla multiorgánica, sepsis y alta mortalidad ${ }^{5,7-11,13-15}$.

\section{Conclusión}

Con sustento en el anterior análisis histórico y técnico, el cadete Agustín Melgar fue víctima de trauma vascular periférico penetrante complejo en la arena militar. Al haber sufrido una condición anatómica tan grave en un ambiente austero y abordado bajo los principios médico-quirúrgicos de la época, sin haber recibido una reanimación adecuada, atención operatoria temprana, analgesia eficaz y manejo de la coagulopatía postraumática, así como por la falta de seguimiento posoperatorio, no sorprende que sucumbiera. Sin embargo, es pertinente señalar que, hoy en día, bajo los protocolos de manejo posmodernos del trauma vascular periférico militar, el pronóstico podría haber cambiado de manera notable y el cadete posiblemente habría sobrevivido, aunque con significativa morbilidad.

\section{Conflicto de intereses}

Los autores de este manuscrito declaran no tener conflicto de intereses.

\section{Agradecimientos y financiamiento}

Agradecimiento a la Lic. Arahí Espino Solís, CEO del CENCAT $^{\circ}$ Curso de Entrenamiento en Cirugía Avanzada en Trauma, por el financiamiento para la elaboración de este manuscrito con fines educativos.

\section{Responsabilidades éticas}

Protección de personas y animales. Los autores declaran que para esta investigación no se han realizado experimentos en seres humanos ni en animales.

Confidencialidad de los datos. Los autores declaran que en este artículo no aparecen datos de pacientes.

Derecho a la privacidad y consentimiento informado. Los autores declaran que en este artículo no aparecen datos de pacientes.

\section{Bibliografía}

1. Departamento del Distrito Federal. El asalto al Castillo de Chapultepec y los Niños Héroes. Colección Conciencia Cívica Nacional. México, D.F.; 1988

2. Bravo UJ. Folletos raros relativos a la Defensa de Chapultepec. Capilla Alfonsina, Fondo Fernando Díaz Ramírez. Ciudad de México; 1848. 
3. Cheselden W. Chap. XXXVIII. Of amputation. En: Sharp S, editor. A treatise on the operations of surgery, with a defcription and reprefentation of the instruments ufed in performing them: to which in prefix'd an introduction on the nature and treatment of wounds, abscesses and ulcers. $3^{\text {rd }}$ ed. London, UK: Brotherton/Innys/Manby/Knapton/Rivington/Lengman/Birt/Hitch/Auften/Hodges \& Hawkins Publishers; 1740. p. 211-25.

4. Dawson DL, Putnam T, Light JL, Inhat DM, Kissinger DP, Rasmussen TE, et al. Temporary arterial shunts to mantain limb perfusion after arterial injury: an animal study. J Trauma. 1999;47:64-71.

5. García-Núñez LM, Cabello-Pasini R, Decuir-Díaz A, Lever-Rosas CD Padilla-Solís R, Gómez-García MA, et al. Lesiones vasculares periféricas complejas y shunts intravasculares temporales. El concepto y la institución del "control de daños" va mucho más allá del abdomen. Rev Sanid Milit Mex. 2005;59:201-7.

6. Moore EE, Malangoni MA, Cogbill TH, Peterson NE, Champion HR Jurkovich GJ, et al. Organ injury scaling VII: cervical vascular, periphera vascular, adrenal, penis, testis, and scrotum. J Trauma. 1996;41:523-4.

7. Asensio JA, Kuncir JE, García-Núñez LM, Petrone P. Femoral vessel injuries: analysis of factors predictive of outcomes. J Am Coll Surg. 2006:203:512-20.

8. Asensio JA, Meneu DJC, Márquez ME, Abradelo UM, Moreno EA García-Núñez LM, et al. Traumatismos penetrantes en extremidades y lesiones vasculares asociadas. En: Asensio JA, Meneu DJC, Moreno GE, editores. Traumatismos. Fisiopatología, diagnóstico y tratamiento. Madrid, España: Jarpyo; 2005. p. 575-84.

9. Asensio JA, Mazzini FN, Pérez-Alonso AJ, Petrone P, Ceballos J, García-Núñez LM, et al. Vascular injuries of the upper and lower extremities. En: Cioffi WG, Asensio JA, Adams CA Jr, Connolly MD, Britt LD, Townsend $\mathrm{CM} \mathrm{Jr}$, et al., editores. Atlas of trauma/emergency surgical techniques. Philadelphia, Pennsylvania, USA: Saunders Elsevier; 2014. p. 270-88.

10. Hernández-García EF, García-Núñez LM. Trauma de extremidades. En: Vega MAJ, Mayagoitia GJC, Ramírez BEJ, Pulido CA, Noyola VHF, editores. Tratado de cirugía general. 3. ${ }^{a}$ ed. Ciudad de México, México: Manual Moderno; 2017. p. 2010-7.

11. García-Núñez LM. Doctor: la pierna está azul... Manejo de las complicaciones del tratamiento del trauma vascular periférico. Ciruj Gen. 2011;33(Suppl 1):S70-2

12. Feliciano DV. Management of peripheral vascular trauma. Disponible en: https://www.facs.org/ /media/files/quality\%20programs/trauma/publications/peripheralvasctrauma.ashx

13. Arcaute VFF, García-Núñez LM, Noyola VHF, Espinoza MF, Rodríguez VCE. Mecanismos de lesión en actos de violencia extrema. Cir Cir. 2016;84:257-62.

14. Nanobashvili J, Kopadze T, Tvaladze M, Buachidze T, Nazvlishvili G. War injuries of major extremity arteries. World J Surg. 2003;27:134-9.
15. Sise MJ, Shackford SR. Extremity vascular trauma. En: Rich NM, Mattox KL, Hirshberg A, editores. Vascular trauma. $2^{\text {nd }}$ ed. Philadelphia, Pennsylvania, USA: Elsevier Saunders; 2004. p. 353-9.

16. Helling TS, McNabney WK. The role of amputation in the management of battlefield casualties: a history of two millennia. J Trauma. 2000;49:930-9.

17. Rish FL, Castañeda GR, Sigler ML. Capítulo 1. Historia del trauma vascular. En: Castañeda GR, Sigler ML, Rish FL, editores. Trauma vascular. Ciudad de México, México: Alfil; 2007. p. 1-15.

18. Rich NM, Rhee P. An historical tour of vascular injury management: from its inception to the new millennium. Surg Clin North Am. 2001;81:1199-216.

19. García-Núñez LM, Asensio JA. Trauma vascular central toracoabdominal. En: Vega MAJ, Mayagoitia GJC, Ramírez BEJ, Pulido CA, Noyola VHF, editores. Tratado de cirugía general. $3 .^{a}$ ed. Ciudad de México, México: Manual Moderno; 2017. p. 1631-9.

20. Asensio JA, Bertelotti R, Mazzini FN, Esparragón CJ, Okwosa C, García-Núñez LM, et al. The syndrome of exsanguination: reliable models to indicate damage control. En: Asensio JA, Trunkey DD, editores. Current therapy of trauma and surgical critical care. $2^{\text {nd }}$ ed. Philadelphia, Pennsylvania, USA: Elsevier; 2016. p. 422-35.

21. Asensio JA, Petrone $P$ O'Shanahan G, Kuncir EJ. Managing exsanguination: what we know about damage control/bail out is not enough. BUMC Proceedings. 2003;16:294-6.

22. Mohr AM, Asensio JA, Karsidag T, García-Núñez LM, Petrone P, Morehouse $A$, et al. Exsanguination: reliable models to indicate damage control. En: Asensio JA, Trunkey DD, editores. Current therapy of trauma and surgical critical care. Philadelphia, Pennsylvania, USA: Elsevier; 2008. p. 282-97.

23. Caprio K, Condon MR, Deitch EA, Xu DZ, Feketova E, Machiedo GW. Alteration of alpha-spectrin ubiquitination after hemorrhagic shock. Am J Surg. 2008;196:663-9.

24. García-Núñez LM, Carreño-Lomelí MA, Muciño-Bolaños MA, Béjar-Cornejo JR, Hernández-García EF. Fístula arterio-venosa iliaca-iliaca inducida por el fenómeno de cavitación temporal en una herida abdominal por proyectil de arma de fuego. Reporte de un caso. Rev Sanid Milit Mex. 2013;67:38-40.

25. García-Núñez LM, Soto-Ortega LE, Delgado-Arámburo JLG, Cabello-Pasini R, Macías-Millan JA. Tratamiento operatorio de las lesiones vasculares carotídeas (Estudio TOLVAC I): cómo abreviar el tiempo de isquemia distal. Rev Sanid Milit Mex. 2009;63:1-8.

26. García-Núñez LM, Soto-Ortega LE, Delgado-Arámburo JLG, Cabello-Pasini R, de la O-García II, Macías-Millan JA, et al. Estudio comparativo de dos técnicas de reparación vascular en trauma penetrante de la arteria carótida, modelo animal. Rev Sanid Milit Mex. 2011;65:241-8. 